\title{
Measurement of the rate of water flow in plants
}

\author{
K. Schurer, H. Griffioen, J. G. Kornet and G. J. W. Visscher \\ Technical and Physical Engineering Research Service (TFDL), \\ Wageningen, the Netherlands
}

Accepted: 17 January 1979

Key words: water flow, stem, thermo-electric

\section{Summary}

A non-destructive thermo-electric method is described for the measurement of water flow in the stems of plants such as wheat, potatoes and tomatoes. The two temperature sensors are 10 or $20 \mathrm{~mm}$ apart. The miniature sensing element is made by evaporation techniques. The sensor is suited for laboratory as well as for field work. Flow rate in the stem can be monitored for weeks at relatively low cost.

\section{Introduction}

The volume of water passing through a cross-section of a plant stem is at any moment nearly equal to the amount lost by evaporation in the plant parts apical of that cross-section. The volume rate can be expressed as the product of the flow rate and the cross-sectional area of the conducting vessels in the stem. If the cross-sectional area is known for a particular type of stem, the flow rate is the only quantity to be measured to get hold of the water balance of the plant.

Measurements of the flow rate in the stems of larger green plants such as cotton, bean and tomato have been reported by Bloodworth et al. $(1955,1956)$. They used a simple non-destructive thermo-electric method.

Other methods are the magnetohydrodynamic technique (Sheriff, 1972), where a magnetic field perpendicular to the flow generates a small voltage in a direction at right angles to both the flow and the field, or the nuclear magnetic resonance technique.

\section{Method}

Heat is applied locally to the stem during a few seconds. The heat flows into the stem in all directions. Part of the heat reaches the xylem vessels and is transported downstream with the moving water. Thus the temperature rise will be higher downstream than it is upstream. Time of occurrence and magnitude of the 
maximum in the temperature difference between points upstream and downstream both depend on the flow rate.

For the highest sensitivity the temperature sensors and the heater should not be too far apart, but their distance should on the other hand be large compared to the depth over which the heat has to travel into the stem to reach the xylem vessels.

The two temperature sensors are placed at equal distances from the heater on a single internodal segment to eliminate effects of direct conduction of heat through the stem tissue.

\section{Design considerations}

The stem should not be affected by the heat. The temperature rise at the heated spot should therefore not exceed a value of approximately $5 \mathrm{~K}$. The flow sensor should be easily placed and replaced on the stem without damage either to the stem or to the sensor. The design should be such as to allow of use of the sensor in the laboratory as well as in the field.

\section{The sensor}

In the first experiments a sensor has been used similar to the ones described by Bloodworth et al. (1955). The dimensions of the sensor, however, were reduced by about a factor of 4 , resulting in a distance between the two thermistors of $10 \mathrm{~mm}$. In the realisation proper positioning and alignment of the thermistors and the heating wire was found very difficult and uncertain. Therefore, an entirely

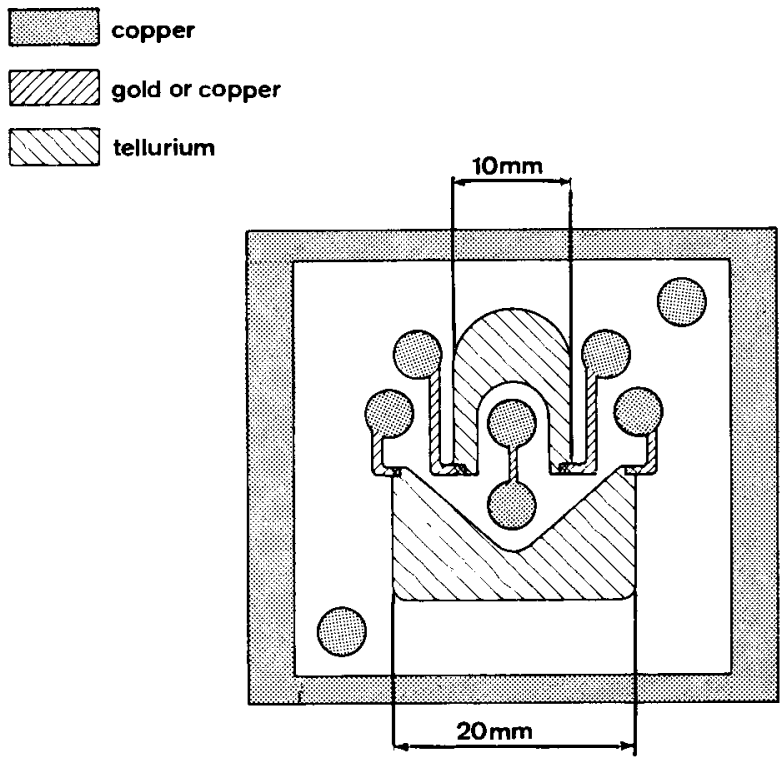

Fig. 1. Evaporated sensing element. 
different design was made by evaporation techniques (Pirani \& Yarwoord, 1961) to produce the temperature sensing and the heating elements. The heating element is a single gold strip, $0.5 \mathrm{~mm}$ wide. The temperature rise is measured with a differential thermocouple consisting of tellurium versus gold. The thermal electromotive force of this couple is about $300 \mu \mathrm{V} / \mathrm{K}$ (Kinzie, 1973). For more flexibility two thermocouples are available on the same substrate, one with the sensors $10 \mathrm{~mm}$ apart and one with the sensors $20 \mathrm{~mm}$ apart.

The sensors can be reproduced to a sufficient precision by the use of masking and photo-etching techniques (Stevens, 1970). The substrate is a flat, copper clad epoxy plate. The copper has been removed except for the six soldering connections (Fig. 1).

The sensor element has an essentially flat surface, thus rendering any damage to the stem unlikely. The surface of the stem, however, can do serious damage to the evaporated metal layers. Several coatings have been tested as a protective layer over the sensor. The most satisfactory results have been obtained with a thin layer of 'Povin' ${ }^{1}$ lacquer, cured for 24 hours at $60{ }^{\circ} \mathrm{C}$. The tellurium layer is affected at higher temperatures, whilst the lacquer remains soft at lower temperatures.

The sensing element is mounted on one leg of a thermally isolating clip. The other leg of the clip bears a $\mathrm{V}$ groove for correct positioning of the sensor on the stem.

\section{Experimental results}

A conservative estimate has been made of the maximum value of the flow rate for which a proportionality between time lapse and flow rate holds. To this end a water flow has been established in a length of plastic tubing with an outer diameter of $4 \mathrm{~mm}$ and a wall thickness of $0.5 \mathrm{~mm}$. Proportionality was within $10 \%$ for flow rates of less than $3 \mathrm{~m} / \mathrm{h}$ and within $5 \%$ for flow rates not exceeding $2 \mathrm{~m} / \mathrm{h}$, both for the narrower thermocouple.

Since the depth over which the heat has to be conducted into the stem of for

1 Supplied by Pope B.V., Venlo, the Netherlands.

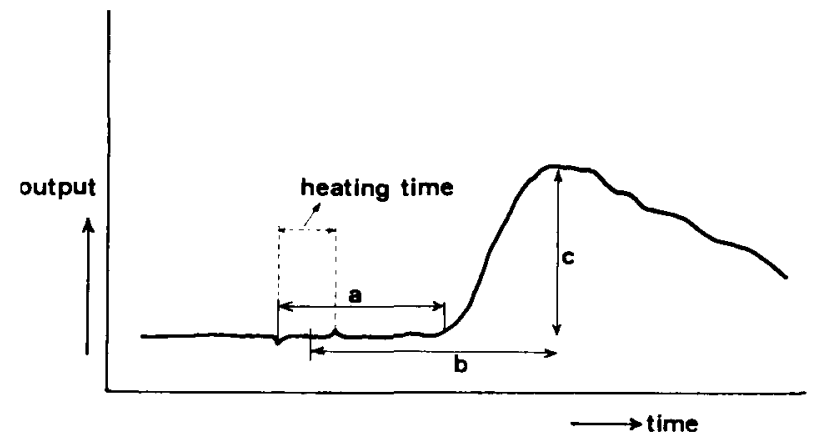

138
Fig. 2. Typical recording of the temperature difference. 


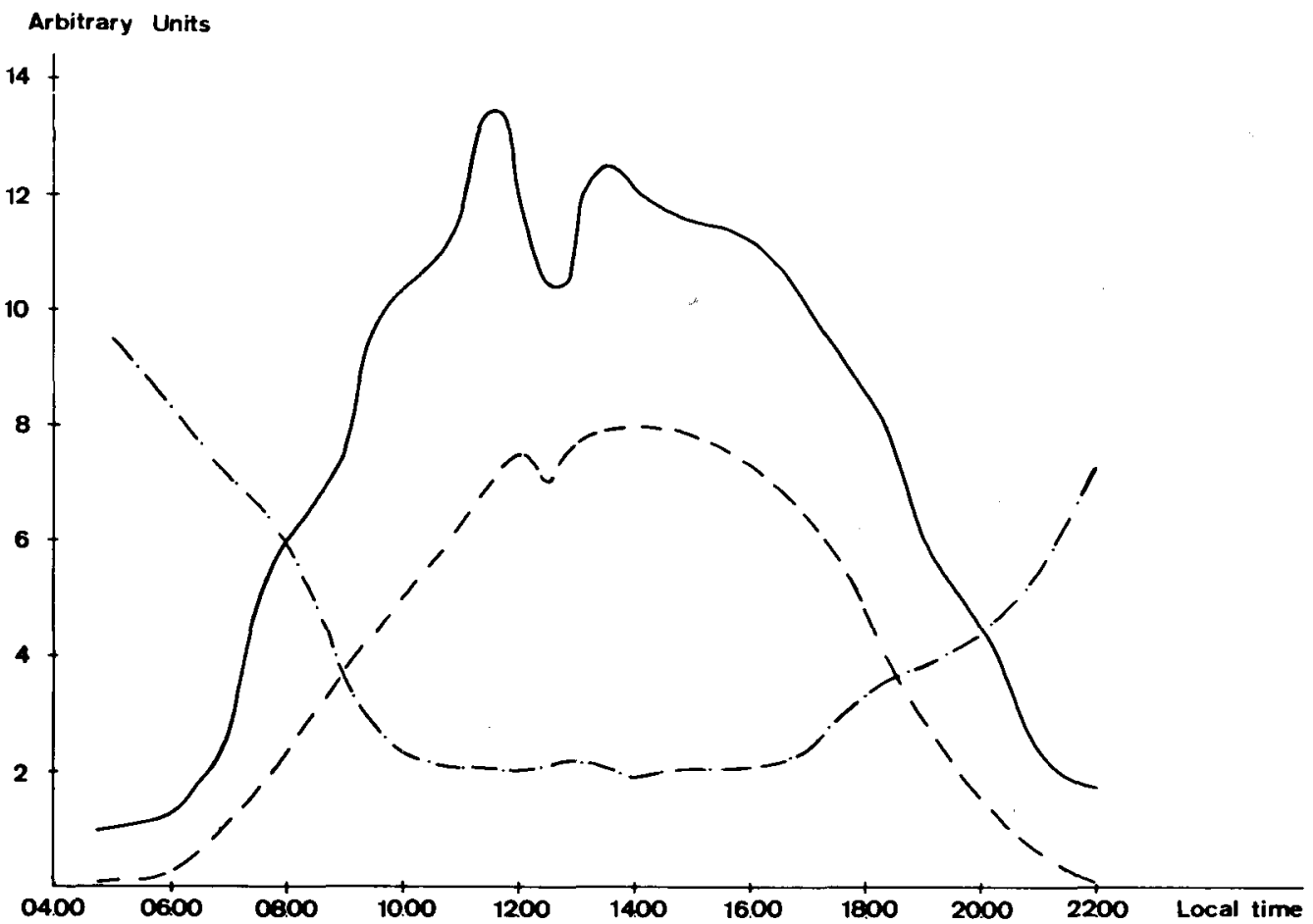

Fig. 3. Diurnal variation of the flow rate in a fully developed potato plant (5 July 1977).

- - - Top height of the output signal.

- . - . - Time lapse between heating and top of the output signal. Irradiation (sunlight).

example a wheat plant is much less, the performance on wheat may be expected to be better than on the plastic tubing.

Experiments have been made on wheat plants in a greenhouse with additional lighting. Reduced flow rates were found when part of the plant was clipped off. The output signal dropped to zero when the plant was clipped off just over the sensor. Comparisons of weight loss in steady-state conditions (Tegelaar \& van der Wal, 1974) with the measured flow rate confirmed the expected proportionality.

The output signal was in the order of $10 \mu \mathrm{V}$. One measurement took one to three minutes, depending on the duration of the heating $(0.5$ to $20 \mathrm{~s})$ and the flow rate. A typical example of a recording is illustrated in Fig. 2.

Field measurements on potato plants (van der Wal, 1978) with five sensors showed most interesting results. An example is given in Fig. 3. Because of the larger depth of the xylem vesels the sensors with a distance of $20 \mathrm{~mm}$ have been used. One of the conclusions was that the instrument offers the possibility of monitoring transpiration patterns of many cultivars in the field. 


\section{Discussion}

Measurements on wheat plants showed, that the top height 'c' (Fig. 2) increases with increasing length of the heating time, and with increasing flow rate. For low flow rates it is difficult to measure the time lapse ' $a$ ' to the beginning of the temperature rise. In that case the time lapse ' $b$ ' is more easily determined. Otherwise ' $a$ ' will give the most precise information on the flow rate.

With the two temperature sensors $10 \mathrm{~mm}$ apart, 'a' showed hardly any variance on wheat during a diurnal period, whilst the top height ' $c$ ' gave very fine results. The measurements on potatoes (van der Wal, 1978) have been made with clips on which the sensors were $20 \mathrm{~mm}$ apart. In this case 'a' turns out to be definitely dependent on flow rate, as well as ' $b$ ' and ' $c$ '.

Attempts to get hold of the phenomenon by means of a theoretical model only yielded qualitative agreements so far.

\section{The electronic circuit}

The output signal of the thermocouple is of the order of 10 to $100 \mu \mathrm{V}$. It is amplified 10000 times by a low-noise amplifier (MP221) to a level suitable for recording and further signal handling. Several thermocouples can be connected to one amplifier over a rotary switch with low thermal electromotive force.

The position of the top of the signal can be determined from the zero passage of its first derivative, or rather, to include drift of the base-line, from the point where the derivative of the signal equals the derivative of the base-line as

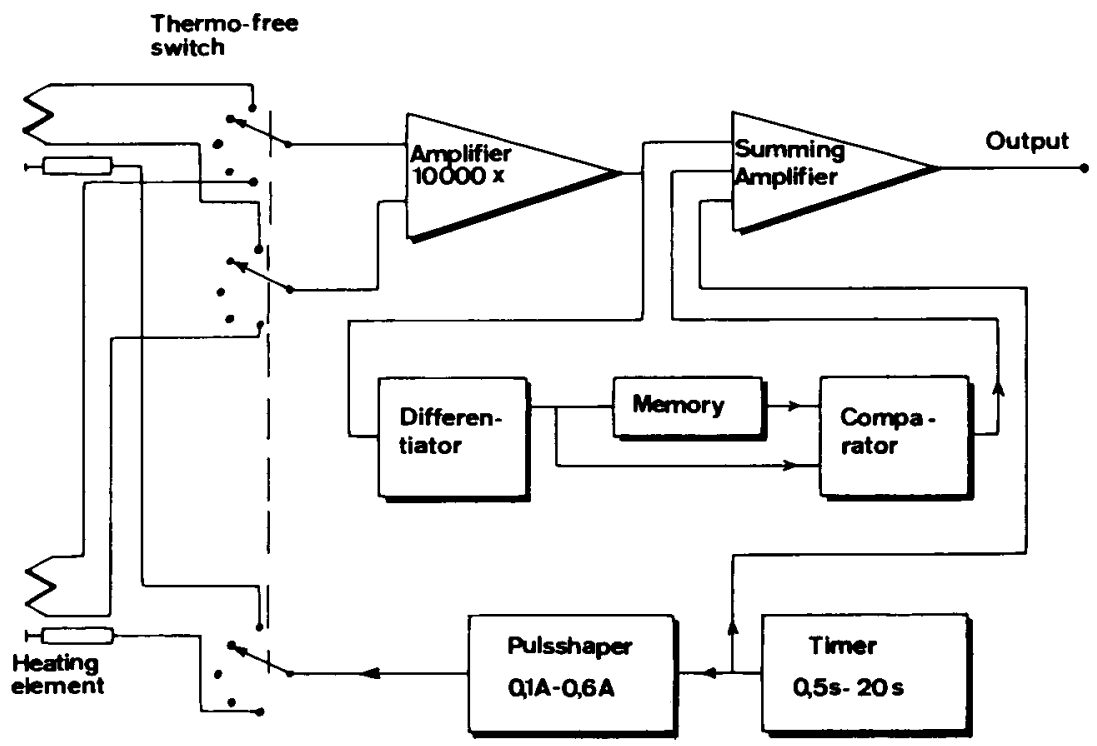

Fig. 4. Block diagram of the electronic circuit. 
measured just before the stem is heated. The actual sequence is then: start procedure (push button); $10 \mathrm{~s}$ measurement of base-line, storage of derivative; heating, indication of start of heating by a peak in the recording; measurement, differentiation indication of top by another peak in the recording; end of measurement. A block diagram of the circuit is shown in Fig. 4.

The settling time after a measurement is in practical situations between five and fifteen minutes, depending on the flow rate.

\section{Acknowledgments}

The authors wish to thank Dr Ir A. F. van der Wal (Foundation for Agricultural Plant Breeding, SVP) and Ir N. M. de Vos (Centre for Agrobiological Research, $\mathrm{CABO}$ ), for initiating the subject and their support during the experiments with the prototype.

\section{References}

Bloodworth, M. E., J. B. Page \& W. R. Cowley, 1955. A thermoelectric method for determining the rate of water movement in plants. Soil Sci. Proc. 19: 411-414.

Bloodworth, M. E., J. B. Page \& W. R. Cowley, 1956. Some applications of the thermoelectric method for measuring water flow rates in plants. Agron. J. 48: 222-226.

Kinzie, P. A., 1973. Thermocouple temperature measurement. Wiley-Interscience, New York, etc.

Pirani, M. \& J. Yarwood, 1961. Principles of vacuum engineering. Chapman \& Hall, London.

Sheriff, D. W., 1972. A new apparatus for the measurement of sap flux in small shoots with the magnetohydrodynamic method. J. exp. Bot. 23: 1086-1095.

Slavík, B., 1974. Methods of studying plant water relations, Ch. 4. Springer Verlag, Berlin, etc.

Stevens, N. B., 1970. In: R. K. Willardson \& A. C. Beer (Ed.), Semiconductors and semimetals, Vol. 5, Infrared detectors. Academic Press, New York/London.

Tegelaar, P. \& A. F. van der Wal, 1974. An ecophysiological approach to crop losses, exemplified in the system wheat, leaf rust and glume blotch. I. A simple and accurate balance for the continuous measuring and recording of evapotranspiration of plants in indoor experiments. Neth. J.Pl. Path. 80: 77-84.

Wal, A. F. van der, 1978. Measuring water flow rate in stems of potato plants in the field. In: Abstracts of conference papers, 7th Triennial Conference of the European Association for Potato Research (Poland, 26 June-1 July), p. 36-37. 